\title{
Assessment of diet quality according to AHEI-2010 in the PURE Poland study
}

\author{
Alicja Basiak-Rasała , Katarzyna Połtyn-Zaradna ${ }^{1}$, Maria Wołyniec ${ }^{1}$, Andrzej Szuba², Katarzyna Zatońska ${ }^{1}$ \\ ${ }^{1}$ Chair and Department of Social Medicine, Wrocław Medical University, Poland \\ ${ }^{2}$ Clinic of Angiology, Hypertension and Diabetology, Wrocław Medical University, Poland
}

\begin{abstract}
Objectives: The Alternate Healthy Eating Index (AHEI) focuses on the elements of nutrition that have the strongest correlation with the risk of chronic diseases. The aim of the analysis was to determine the quality of the diet according to the AHEI score of participants of PURE Poland cohort and to ascertain whether there was an association between AHEI score and risk of cardiometabolic syndrome (CardMetS).

Material and methods: The Polish cohort of the PURE study was established in 2007 in Wrockaw. The population of the analysis consists of 2026 participants aged between 30 and 85 years. The AHEI score was calculated based on the Food Frequency Questionnaire (FFQ). CardMetS was diagnosed with at least three out of the following five symptoms: elevated blood glucose, elevated blood pressure, low HDL, high triglycerides, and abdominal obesity. Statistical significance was stated when $p<0.05$.

Results: The average AHEI score of the participants was 46.66. Participants living in rural areas had significantly $(p<0.05)$ lower AHEI score of the diet than those living in urban areas (mean: $44.01 \mathrm{vs.}$ 48.48). Men had significantly ( $p<0.05$ ) lower AHEI score than women (mean 45.64 vs. 47.25 ). There was weak but significant positive correlation between the AHEI score and age of participants (correlation of 0.1 ) and with level of education (correlation of 0.15). Participants with CardMetS had significantly lower AHEI score of the diet than participants without CardMetS $(p=0.042)$.

Conclusions: Programs of prevention should be provided mainly for middle aged men, living in rural areas, with lower level of education. Higher AHEI score of the diet was associated with lower risk of cardiometabolic syndrome.
\end{abstract}

KEY WORDS: diet, PURE study, AHEI.

ADDRESS FOR CORRESPONDENCE: Alicja Basiak-Rasała, Chair and Department of Social Medicine, Wrocław Medical University, 44 O. Bujwida St., 50-345 Wrocław, e-mail: alicja.basiak-rasala@umed.wroc.pl

\section{INTRODUCTION}

Noncommunicable diseases (NCDs), including cardiovascular diseases (CVD), cancer, diabetes, and chronic respiratory diseases, are a leading cause of death worldwide [1]. Most of the mortality related to NCDs is premature and can be delayed. According to the World Health Organization (WHO) tobacco smoking, unhealthy diet, insufficient physical activity, and harmful use of alcohol are leading modifiable risk factors of NCDs [1]. Balanced nutrition is one of the key elements of maintaining health. Nutrition-related NCDs, as well as overweight and obesity, are becoming more and more prevalent not only in adults but also in children and adolescents [2]. The western type of diet, characterised by higher consumption of red and processed meat, refined grains, and full-fat dairy products is associated with higher risk of CVD and other NCDs [3]. On the other hand, increased consumption of vegetables, fruit, and legumes [4], whole grains [5] and higher adherence to Mediterranean diet guidelines [6] are factors associated with decreased risk of cardiovascular diseases, cancer, and total mortality. Worldwide, the consumption of vegetables, fruit, nuts/seeds, whole grains, and fish is lower than recommended in dietary guidelines [7]. 
Over the years, several indices have been created to enable the assessment of the quality of the diet. The Alternate Healthy Eating Index (AHEI) was created as a modification of the Healthy Eating Index (HEI) in order to focus on the elements of nutrition that have the strongest correlation with the risk of chronic diseases [8]. The HEI score was created to assess the adherence to the United States Department of Agriculture (USDA) nutritional guidelines for the general population; the higher the AHEI score, the lower the risk of cardiovascular diseases and cardiovascular deaths, heart failure, diabetes, colorectal cancer, and breast cancer [8]. According to a meta-analysis conducted by Schwingshackl et al. [9], people who had higher quality of diet, expressed also in higher AHEI score, had lower risk of all-cause mortality, CVD, cancer, type 2 diabetes, and neurodegenerative diseases. In the study by Wu et al., participants with higher AHEI score of the diet also had significantly lower serum low-density lipoprotein cholesterol level and lower waist circumference [10]. Moreover, higher AHEI score was associated with lower body mass, lower waist-to-height ratio, moderate and vigorous activity, and reduced sedentary behaviour [11].

Cardiometabolic syndrome (CardMetS) was associated with increased risk of CVD events [12]. CardMetS was associated with a two-fold increase in the risk of cardiovascular diseases and a four-fold increase in the risk of diabetes [13]. According to recent consensus, CardMetS is diagnosed with occurrence of at least three out of five of the following symptoms: elevated blood glucose, elevated blood pressure, low HDL cholesterol, high triglycerides, and abdominal obesity [13]. Approximately $1 / 5$ of the Polish population had CardMetS [14] in comparison to average $1 / 4$ of the European population (23.9\% in men and $24.6 \%$ in women) [15].

The aim of the analysis was to determine the quality of the diet according to AHEI score of participants of the PURE Poland cohort and determine the sociodemographic profile of participants at higher risk of developing NCDs according to AHEI score. The secondary aim was to ascertain if there was an association between AHEI score values and risk of CardMetS in the PURE Poland cohort.

\section{MATERIAL AND METHODS}

Poland is one of the 21 countries enrolled in the global Prospective Urban and Rural Epidemiology Study (PURE). All participants were examined according to the PURE project protocol, which has been described in details elsewhere $[16,17]$. The PURE study aims to assess the impact of different risk factors (socioeconomic, environmental, behavioural, and biological) on the incidence of NCDs. The PURE study scope and data collection are presented in Figure 1. The study was designed to collect data in three-year intervals. Currently, the 12-year follow-up has just started. The Polish cohort of the PURE study was established in 2007 in Wrocław (Lower Silesia province). The baseline cohort, enrolled between 2007 and 2010, included 2036 participants, aged between 30 and 85 years, both from urban and rural areas. The general protocol included the following questionnaires: household, family, individual health questionnaires, the International Physical Activity Questionnaire (IPAQ), and the Food Frequency Questionnaire (FFQ). Moreover, following examinations were performed: anthropometric measurements (including body height, body mass, waist and hip circumference), spirometry, ECG, and blood pressure measurement. Biological material (blood, urine) was collected from all participants. The population of the analysis herein consisted of 2026 participants aged between 30 and 85 years, who completed FFQ at baseline (10 participants were excluded due to lack of dietary data). The FFQ consisted of 154 products that are characteristic of Polish nutritional habits. The FFQ was collected by trained staff and referred to participants' dietary habits one year prior to the interview. The FFQ was specially developed and validated for the Polish population [18]. To assess the daily intake of each food product from the FFQ, the frequency of consumption reported by participants was multiplied by serving size. Subsequently, food intake was converted into macro- and micronutrient intake based on food composition tables [18]. Participants whose calorie intake assessed by FFQ was lower than $500 \mathrm{kcal} / \mathrm{d}$ or higher than 5000 $\mathrm{kcal} / \mathrm{d}$ were excluded from the analysis due to unreliable interview. AHEI-2010 score was calculated according to methodology described by Chiuve et al. [8]. Total AHEI score was the sum of scores received in 10 dietary categories (we excluded trans unsaturated fatty acids due to lack of data): 1) vegetables, 2) fruit, 3) whole grains, 4) sugar-sweetened beverages (SSB) and fruit juice, 5) nuts and legumes, 6) red and processed meat, 7) long chain (n-3) polyunsaturated fatty acids (PUFA) (EPA + DHA), 8) total PUFA intake, 9) sodium, and 10) alcohol. In every nutritional category participants could receive between 0 (worst) and 10 (best) points. The rationale for selection of those particular elements of diet and scoring system for every category was described by Chiuve et al. [8]. The total AHEI score could range from 0 (worst) to 100 points (best). The scoring system is presented in Table 1. In the case of n-3 PUFA score, we did not have data regarding the exact EPA + DHA intake from the FFQ, so we estimated it based on fish intake following calculation guidelines (recommended $250 \mathrm{mg} / \mathrm{d}$ of EPA + DHA equals on average 2-4 ounces of fish per week) [8].

The CardMetS status was defined using the interim statement [13] as having at least three of the following criteria: abdominal obesity (waist circumference $\geq 94 \mathrm{~cm}$ for men and $\geq 80 \mathrm{~cm}$ for women), high blood pressure (BP) (SBP/DBP $\geq 130 / 85 \mathrm{~mm} \mathrm{Hg}$ or antihypertensive medication), hypertriglyceridaemia $(\geq 150 \mathrm{mg} / \mathrm{dl}$ or antihypertriglyceridaemia medication), low HDL-cho- 


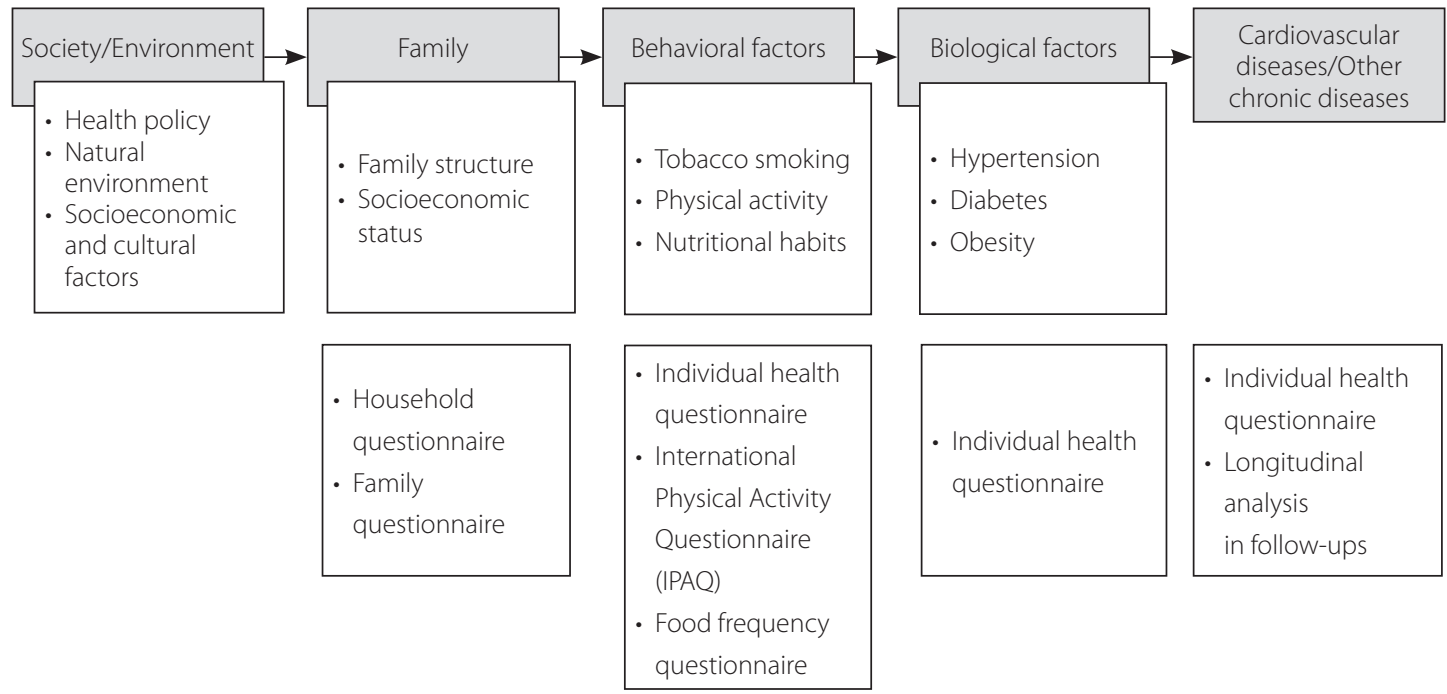

FIG. 1. PURE study scope and design (based on [16])

lesterolaemia $(<40 \mathrm{mg} / \mathrm{dl}$ for men or $<50 \mathrm{mg} / \mathrm{dl}$ for women), and hyperglycaemia (fasting blood glucose $\geq 100 \mathrm{mg} / \mathrm{dl}$ or antidiabetic medication).

Statistical analysis of association between AHEI score and sex and place of residence was calculated with the use of a $t$-test. To assess the association between AHEI, age, and level of education Pearson's linear correlation was used $(p<0.05)$. To assess the association between AHEI score and CardMets, Wilcoxon rank sum test was implemented.

Written, informed consent was obtained from all participants enrolled in the study. All human studies were reviewed by the appropriate ethics committee and have therefore been performed in accordance with the ethical standards laid down in an appropriate version of the 1964 Declaration of Helsinki (positive opinion of The Bioethics Committee of Wrocław Medical University no. KB-443/2006).

\section{RESULTS}

The average AHEI score of the participants was 46.66 . $39.49 \%$ of participants had CardMets. Participants living in rural areas had significantly $(p<0.05)$ lower AHEI score of the diet than those living in urban areas (mean: 44.01 vs. 48.48$)$. Men had significantly $(p<0.05)$ lower AHEI score than women (mean 45.64 vs. 47.25). There was weak but significant positive correlation between the AHEI score and age of participants (correlation of 0.1), as well as with level of education (correlation of 0.15). Marital status was not a significant factor differentiating risk of developing chronic diseases according to AHEI2010 score.

Considering AHEI score components, on average the highest score was observed in nuts/legumes (8.10), sodium (7.74), and fish (6.69). The lowest values were observed in red/processed meat (1.21), alcohol (3.46), and sugar-sweetened beverages (SSB) (4.13) (Fig. 2).
When comparing AHEI components between urban and rural participants (Fig. 3), rural dwellers were characterised by lower values in almost every category except PUFA and fish. The most noticeable differences in scores between urban and rural participants were observed in SSB (4.97 vs. 2.90 , respectively), red and processed meat ( 1.73 vs. 0.45 , respectively), vegetables ( 4.83 vs. 3.98 , respectively), and fruit (5.78 vs. 4.73 , respectively). Differences in scores obtained by men and women were less significant (Fig. 4). Noticeable differences were seen in scores for vegetables (3.95 vs. 4.79, respectively), fruit (4.59 vs. 5.79 , respectively), and alcohol (4.37 vs. 2.92 , respectively).

Participants with CardMetS had significantly lower AHEI score of the diet than participants without CardMetS $(p=0.042)$. Average AHEI score of participants with CardMets was 46.27 and of healthy participants it was 47.08 .

\section{DISCUSSION}

In the paper herein we assessed the quality of diet with the use of the AHEI score and sociodemographic characteristics of the population related to AHEI score. In our cohort, lower AHEI score was significantly associated with the risk of CardMetS. Rural dwellers had significantly lower AHEI score of the diet than urban dwellers. Moreover, men had significantly lower diet quality according to AHEI score than women.

Higher quality of the diet has been already associated with better anthropometric markers of cardiometabolic health, such as lower waist circumference and body mass index [19]. In a cohort study by Mattei et al. [20] higher diet quality expressed by higher AHEI values was associated with higher risk of CardMetS, but the association varied by ethnic background. The same authors observed that minor improvements in diet quality resulted in major cardiometabolic risk reduction. Each 


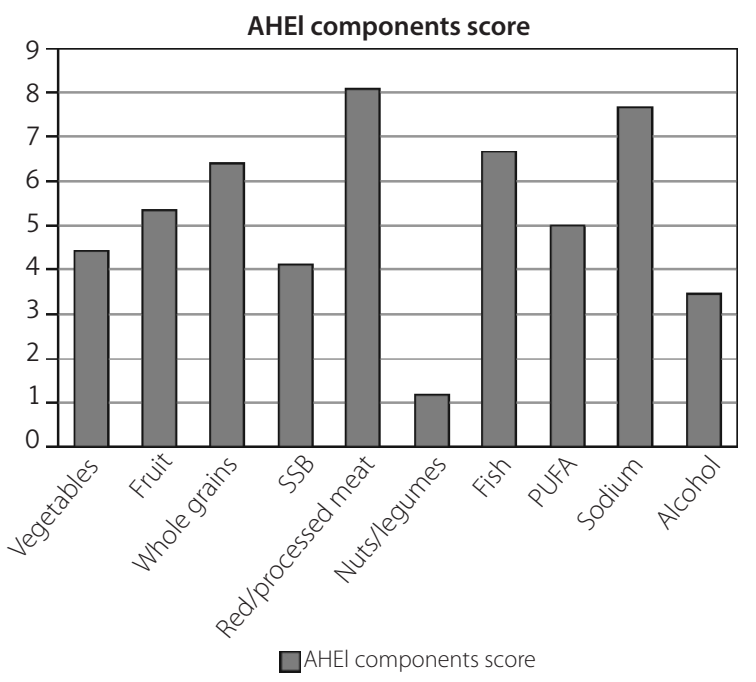

FIG. 2. AHEl components score in overall study population (SSB - sugar sweetened-beverages, PUFA - polyunsaturated fatty acids)

additional 10-unit increase in AHEI was associated with 22\% lower CardMetS risk [20]. Some authors observed that AHEI score had a stronger association with CVD and diabetes in comparison to other diet quality scores $[8,21]$. Diet quality scores, like AHEI, were associated not only with anthropometric markers of health but also with inflammatory biomarkers. AHEI score was inversely associated with serum concentration of leptin, CRP [22], and insulin [23].

Rural populations were usually characterised by higher prevalence of cardiometabolic risk factors, including worse diet quality than in urban populations $[24,25]$. Similarly, in our cohort, diet quality expressed in AHEI score was lower in rural populations than in urban. According to our previous research [25], the diet of women and residents of urban areas were closer to recommendations regarding consumption of energy and macronutrients in comparison to men and residents of urban areas. In the analysis herein, according to AHEI score, the rural population was characterised by lower consumption of fruit and vegetables and higher consumption of SSBs and red/processed meat. Such a dietary pattern is highly unfavourable and can increase the risk of several chronic diseases. The WHO recommends consumption of at least $400 \mathrm{~g}$ of fresh fruit and vegetables per day to maintain health [26]. Higher consumption of fruit, vegetables, and legumes has been associated with lower mortality in the global PURE study [4]. Lower consumption of fruit and vegetables seems also to be inversely associated with risk of CardMetS [27]. According to Guasch-Ferré et al., replacing red and processed meat with high-quality plant protein sources results in lower level of blood lipids and lipoproteins, decreasing the risk of cardiovascular diseases [28]. Consumption of red and processed meat has been also associated with higher risk of type 2 diabetes [29] and some types of cancer [30].

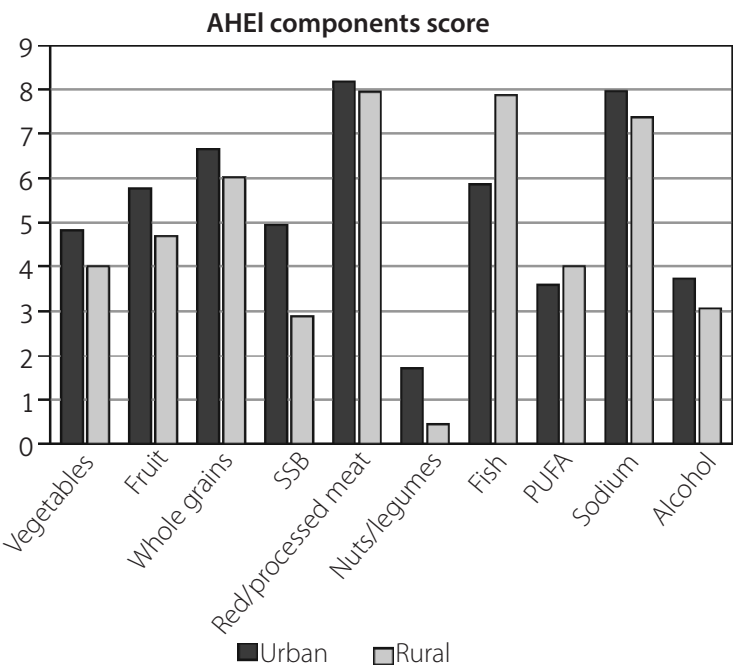

FIG. 3. Comparison of AHEI components score between urban and rural place of residence (SSB - sugar sweetened-beverages, PUFA - polyunsaturated fatty acids)

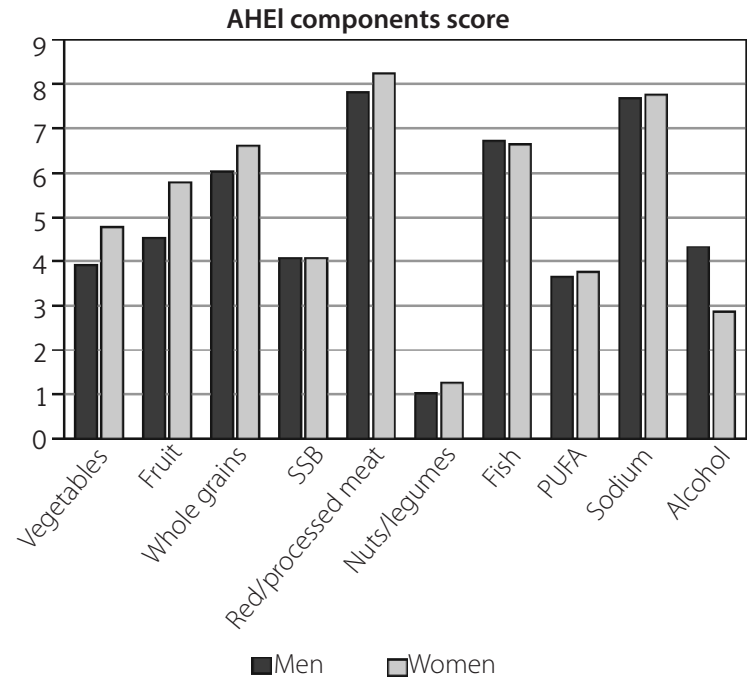

FIG. 4. Comparison of AHEl components score between men and women) (SSB - sugar sweetened-beverages, PUFA - polyunsaturated fatty acids)

In our analysis, lower level of education was associated with lower AHEI score of the diet. This observation is consistent with results from other studies [31]. In our study men had significantly lower total AHEI score of the diet than women. Our finding is consistent with other studies. According to global analyses, men tend to have lower quality of the diet than women [32]. Interestingly, in our cohort women had lower scores for alcohol consumption on average than men. Those results can be explained by the scoring method used for AHEI. According to AHEI scoring methodology, moderate amounts of alcohol may be consumed as part of a healthy diet and therefore receive a higher score than non-drinkers [8]. The influence of alcohol consumption on health and allowed dosages are the subject of wide public debate. Recently published results from the Global Burden of 
Diseases Study 2016 indicate that no amount of alcohol can be perceived as safe [33]. The authors concluded that all-cause mortality increases with increasing consumption of alcohol and suggested a more restrictive approach in developing health policies.

There are some limitations to consider. FFQ assesses the intake of nutritional products retrospectively, which can contribute to overestimation of consumption of recommended dietary products and underestimation of consumption of unhealthy products. Thus, the quality of the diet expressed by AHEI score can be slightly overestimated. On the other hand, a strength of our study is that FFQ was specially developed and validated for a regional population [18], which improves the credibility of dietary results. A further limitation regards the time of data collection; because the baseline study data was collected between 2007 and 2010, it is possible that overall diet quality changed since then, and this fact should be taken into consideration in any comparison of the results with those of other studies.

\section{CONCLUSIONS}

Dietary counselling in prevention of chronic diseases should be provided predominantly for middle aged men, living in rural areas, with lower level of education. Diet quality expressed in higher AHEI score was associated with lower risk of cardiometabolic syndrome.

\section{DISCLOSURE}

The authors report no conflict of interest.

\section{References}

1. World Health Organization. Global status report on noncommunicable diseases 2010. World Health Organization, Geneva 2015.

2. United States Department of Agriculture and Department of Health and Human Services. 2015-2020 Dietary Guidelines for Americans. Washington, 2015. doi: 10.1097/NT.0b013e31826c50af.

3. Hosseini Z, Whiting SJ, Vatanparast H. Current evidence on the association of the metabolic syndrome and dietary patterns in a global perspective. Nutr Res Rev 2016; 29 (2): 152-162.

4. Miller V, Mente A, Dehghan M, et al. Fruit, vegetable, and legume intake, and cardiovascular disease and deaths in 18 countries (PURE): a prospective cohort study. Lancet 2017; 390 (10107): 2037-2049.

5. Aune D, Keum N, Giovannucci E, et al. Whole grain consumption and risk of cardiovascular disease, cancer, and all cause and cause specific mortality: systematic review and dose-response meta-analysis of prospective studies. BMJ 2016; 353: i2716.

6. Estruch R, Ros E, Salas-Salvadó J, et al. Primary prevention of cardiovascular disease with a mediterranean diet supplemented with extra-virgin olive oil or nuts. N Engl J Med 2018; 378 (25): e34.

7. Micha R, Khatibzadeh S, Shi P, et al. Global, regional and national consumption of major food groups in 1990 and 2010: a systematic analysis including 266 country-specific nutrition surveys worldwide. BMJ Open 2015; 5 (9): e008705.

8. Chiuve SE, Fung TT, Rimm EB, et al. Alternative dietary indices both strongly predict risk of chronic disease. J Nutr 2012; 142 (6): 1009-1018.

9. Schwingshackl L, Bogensberger B, Hoffmann G. Diet quality as assessed by the healthy eating index, alternate healthy eating index, dietary approaches to stop hypertension score, and health outcomes: an updated systematic review and meta-analysis of cohort studies. J Acad Nutr Diet 2018; 118 (1): 74-100.

10. Wu PY, Huang CL, Lei WS, Yang SH. Alternative health eating index and the Dietary Guidelines from American Diabetes Association both may reduce the risk of cardiovascular disease in type 2 diabetes patients. J Hum Nutr Diet 2016; 29 (3): 363-373.

11. Fallaize R, Livingstone KM, Celis-Morales $\mathrm{C}$, et al. Association between diet-quality scores, adiposity, total cholesterol and markers of nutritional status in european adults: Findings from the Food4Me study. Nutrients 2018; 10 (1). pii: E49.

12. Scuteri A, Najjar SS, Morrell CH, et al. The metabolic syndrome in older individuals: prevalence and prediction of cardiovascular events: the Cardiovascular Health Study. Diabetes Care 2005; 28 (4): $882-887$.

13. Alberti KG, Eckel RH, Grundy SM, et al. Harmonizing the metabolic syndrome: a joint interim statement of the International Diabetes Federation Task Force on Epidemiology and Prevention; National Heart, Lung, and Blood Institute; American Heart Association; World Heart Federation; International Atherosclerosis Society; and International Association for the Study of Obesity. Circulation 2009; 120 (16): 1640-1645.

14. Drygas W, Bielecki W, Kozakiewicz K, et al. Wieloośrodkowe Ogólnopolskie Badanie Stanu Zdrowia Ludności - WOBASZ. [National Survey on Health Status]. Med Prakt 2015.

15. Scuteri A, Laurent S, Cucca F, et al. Metabolic syndrome across Europe: different clusters of risk factors. Eur J Prev Cardiol 2015; 22 (4): 486-491.

16. Teo K, Chow CK, Vaz M, et al. The Prospective Urban Rural Epidemiology (PURE) study: examining the impact of societal influences on chronic noncommunicable diseases in low-, middle-, and high-income countries. Am Heart J 2009; 158 (1): 1-7.e1.

17. Zatońska K, Zatoński WA, Szuba A. Prospective urban and rural epidemiology Poland - study design. J Health Inequal 2016; 2 (2): 136-141.

18. Dehghan M, Ilow R, Zatonska K, et al. Development, reproducibility and validity of the food frequency questionnaire in the Poland arm of the Prospective Urban and Rural Epidemiological (PURE) study. J Hum Nutr Diet 2012; 25 (3): 225-232.

19. Livingstone KM, McNaughton SA. Association between diet quality, dietary patterns and cardiometabolic health in Australian adults: a cross-sectional study. Nutr J 2018; 17 (1): 19.

20. Mattei J, Sotres-Alvarez D, Daviglus ML, et al. Diet quality and its association with cardiometabolic risk factors vary by hispanic and latino ethnic background in the hispanic community health study/ study of Latinos. J Nutr 2016; 146 (10): 2035-2044. 
21. Belin RJ, Greenland P, Allison M, et al. Diet quality and the risk of cardiovascular disease: the Women's Health Initiative (WHI). Am J Clin Nutr 2011; 94 (1): 49-57.

22. Ko BJ, Park KH, Shin S, et al. Diet quality and diet patterns in relation to circulating cardiometabolic biomarkers. Clin Nutr 2016; 35 (2): 484-490.

23. AlEssa HB, Malik VS, Yuan C, et al. Dietary patterns and cardiometabolic and endocrine plasma biomarkers in US women. Am J Clin Nutr 2017; 105 (2): 432-441.

24. Trivedi T, Liu J, Probst J, et al. Obesity and obesity-related behaviors among rural and urban adults in the USA. Rural Remote Health 2015; 15 (4): 3267.

25. Różańska D, Regulska-Ilow B, Ilow R, et al. Differences in energy and selected macronutrients intake with diet between men and women from urban and rural areas of the Lower Silesia in Poland : the Polish arm of the Prospective Urban Rural Epidemiological (PURE) study. J Heal Inequal 2016; 2 (2): 148-154.

26. Bazzano LA, Israel B. Dietary Intake of fruit and vegetables and risk of diabetes mellitus and cardiovascular diseases. World Health Organization, Geneva 2005.

27. Lee M, Lim M, Kim J. Fruit and vegetable consumption and the metabolic syndrome: a systematic review and dose-response meta-analysis. Br J Nutr 2019; 13: 1-11.

28. Guasch-Ferré M, Satija A, Blondin SA, et al. Meta-analysis of randomized controlled trials of red meat consumption in comparison with various comparison diets on cardiovascular risk factors. Circulation 2019; 139 (15): 1828-1845.

29. Feskens EJ, Sluik D, van Woudenbergh GJ. Meat consumption, diabetes, and its complications. Curr Diab Rep 2013; 13 (2): 298306.

30. Bouvard V, Loomis D, Guyton KZ, et al. Carcinogenicity of consumption of red and processed meat. Lancet Oncol 2015; 16 (16): 1599-1600

31. Huffman FG, De La Cera M, Vaccaro JA, et al. Healthy eating index and alternate healthy eating index among Haitian Americans and African Americans with and without type 2 diabetes. J Nutr Metab 2011; 2011: 398324

32. Imamura F, Micha R, Khatibzadeh S, et al. Dietary quality among men and women in 187 countries in 1990 and 2010: a systematic assessment. Lancet Glob Health 2015; 3 (3): e132-142.

33. GBD 2016 Alcohol Collaborators. Alcohol use and burden for 195 countries and territories, 1990-2016: a systematic analysis for the Global Burden of Disease Study 2016. Lancet 2018; 392 (10152): 1015-1035. 